\title{
Article
}

\section{Self-Assembled Lipid Cubic Phase and Cubosomes for the Delivery of Aspirin as a Model Drug}

Kulkarni, Chandrashekhar Vishwanath, Vishwapathi, Vinod, Quarshie, Abraham, Moinuddin, Zeinab, Page, James, Kendrekar, Pravin and Mashele, Samson S.

Available at https://clok.uclan.ac.uk/20262/

Kulkarni, Chandrashekhar Vishwanath orcid iconORCID: 0000-0002-56214791, Vishwapathi, Vinod, Quarshie, Abraham, Moinuddin, Zeinab, Page, James, Kendrekar, Pravin and Mashele, Samson S. (2017) Self-Assembled Lipid Cubic Phase and Cubosomes for the Delivery of Aspirin as a Model Drug. Langmuir, 33 (38). pp. 9907-9915. ISSN 0743-7463

It is advisable to refer to the publisher's version if you intend to cite from the work. http://dx.doi.org/10.1021/acs.langmuir. 7 b02486

For more information about UCLan's research in this area go to http://www.uclan.ac.uk/researchgroups/ and search for <name of research Group>.

For information about Research generally at UCLan please go to http://www.uclan.ac.uk/research/

All outputs in CLoK are protected by Intellectual Property Rights law, including Copyright law. Copyright, IPR and Moral Rights for the works on this site are retained by the individual authors and/or other copyright owners. Terms and conditions for use of this material are defined in the policies page. 


\title{
Self-Assembled Lipid Cubic Phase and Cubosomes for the Delivery of a Model Drug (Aspirin)
}

\author{
Chandrashekhar V. Kulkarni $^{a *}$, Vinod Kumar Vishwapathi ${ }^{a, b}$, Abraham Quarshie ${ }^{a}$, \\ Zeinab Moinuddin ${ }^{a}$, James Page ${ }^{a}$, Pravin Kendrekar ${ }^{c}$ and Samson S. Mashele ${ }^{c}$ \\ a School of Physical Sciences and Computing, University of Central Lancashire, Preston PR1 \\ 2HE, United Kingdom \\ ${ }^{b}$ School of Pharmacy and Biomedical Sciences, University of Central Lancashire, Preston PR1 \\ 2HE, United Kingdom \\ ${ }^{c}$ Unit for Drug Discovery Research, Faculty of Health and Environmental Sciences, Central \\ University of Technology (CUT), Bloemfontein 9300, Free State, South Africa
}

*Corresponding author email: cvkulkarni@uclan.ac.uk, Tel: +44-1772-89-4339, Fax: +441772-89-4981.

KEYWORDS: lipid cubic phase, cubosomes, drug delivery, lipid nanoparticles, sustained release, drug nanocarriers, nanomedicine.

\begin{abstract}
Three-dimensionally organized lipid cubic self-assemblies and derived oil-in-water emulsions called 'cubosomes' are attractive for various biotechnological applications due to their ability to be loaded with functional molecules and associated sustained release properties. Here we employed both lipid-based systems for the delivery of a model drug - aspirin under comparable conditions. Studies were performed by varying drug to lipid ratio and the type of release medium, water and phosphate buffer saline (PBS). Release rates were determined using UVVis spectroscopy while small angle X-ray scattering confirmed the type of self-assembled nanostructures in lipid systems. The release from bulk lipid cubic phase was sustained as compared to the dispersed cubosomes while the release in PBS was efficient than in water. Highly tortuous architecture, length of the diffusion pathway, type of nanostructure and physicochemical interaction with the release media evidently contribute to these observations. This work is particularly important, as it is the first report where both of the nanostructured lipid systems were studied together under similar conditions. The work provides important insights in understanding, and therefore controlling the release behaviour of lipid-based drug nanocarriers.
\end{abstract}




\section{Introduction}

Lipids, generally composed of hydrophilic and hydrophobic molecular components (Figure 1a), tend to self-assemble in presence of aqueous media. Self-assemblies can be as simple as spherical micelles and planar bilayers or they can be quite elegant like hexagonal and cubic phases $^{1-2}$. Based on the spatial organisation, lipid cubic phases are divided into two types ${ }^{3}$, bicontinuous and micellar ${ }^{3}$. Most common bicontinuous cubic phases (Figure 1b), defined by crystallographic space groups Ia3d (no. 230), Pn3m (no. 224) and Im3m (no. 229), are formed by draping a continuous lipid bilayer on Gyroid (G), diamond (D) and primitive (P) type periodic minimal surfaces, respectively ${ }^{3-4}$. The term 'bicontinuous' can be interpreted in two possible ways, 1) continuity of two networks- first made of a continuous bilayer and second made of continuous waterways, and 2) presence of two continuous aqueous channels separated by a single lipid bilayer ${ }^{5}$. Micellar cubic phases, on the other hand, are formed by an arrangement of discrete micelles in a cubic lattice ${ }^{3}$. Typical example of a micellar cubic phase is $F d 3 m$ phase ${ }^{6}$.

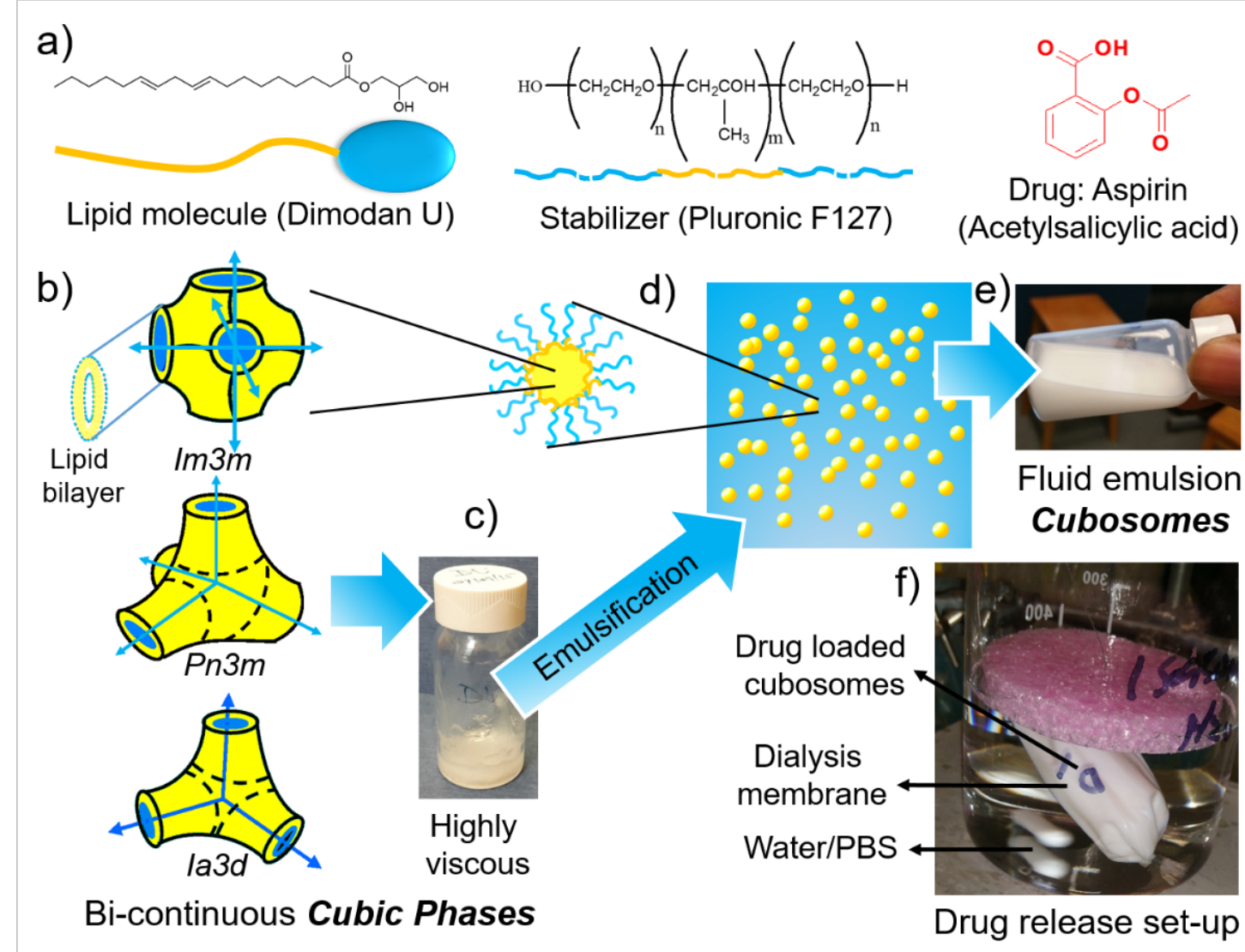

Figure 1: Schematic diagram depicting cubic phases, cubosomes and release set-up (not on scale): a) Chemical structures of a lipid molecule, a surfactant stabilizer and a drug. Blue and yellow colour shades code for hydrophilic and hydrophobic parts/regions, respectively. b) 
Bicontinuous cubic phases of $\operatorname{Im} 3 m, P n 3 m$ and Ia3d types with corresponding 6, 4 and 3 aqueous channels as represented by arrows. c) Highly viscous cubic phase (Pn3m in the current study) is shown at the bottom of $20 \mathrm{ml}$ glass bottle. d) Graphics of cubosome on the left displays Im3m phase as an internal self-assembly while the surfactant molecules stabilize the interface; dispersion of such cubosomes is shown on the right. e) Cubosome dispersion is essentially a form of an oil-in-water emulsion, which exhibits fluid and milky consistency. f) A beaker containing water/PBS with a dialysis membrane holding drug-loaded cubosomes. It represents a typical drug release set-up employed in the current work.

Bicontinuous cubic phases are equipped with a range of structural features responsible for their applicability; for instance, a) enormous surface area $\left(400 \mathrm{~m}^{2} / \mathrm{g}\right)^{7}$ b) large surface to volume ratio c) physicochemical ability to load hydrophilic molecules in aqueous region, hydrophobic molecules in lipid chain region and amphiphilic molecules in bilayers ${ }^{8}$ d) continuous trajectories for uninterrupted diffusion of loaded molecules ${ }^{5}$ e) viscoelastic nature for the stability and structural integrity of loaded molecules ${ }^{9} \mathrm{f}$ ) highly organized porous network for nanoscale templating ${ }^{10}$ and g) resemblance with biomembrane structures ${ }^{11}$. Thermodynamic equilibrium ${ }^{12}$ and robustness under given conditions, and high level of tunability are further aspects. However, the preparation and the handling of cubic phases are not simple protocols due to inherent high viscosity (complex viscosity in the range of $10^{4}-10^{5}$ pa.s) ${ }^{13}$ (Figure $1 \mathrm{c}$ ). Two possible ways to overcome these hurdles are, to utilize mechanical tools to handle cubic phases $^{14-16}$ or to disperse them into a fluid form ${ }^{17-18}$ (Figure $1 \mathrm{~d}, \mathrm{e}$ ). The latter means provides additional benefits, for instance, improved surface to volume ratio ${ }^{19}$, availability of enormous aqueous reservoir (up to $95-96 \%$ of the volume) ${ }^{20-21}$, rather simple preparation protocols, low overall viscosity and rather regular domains (monodispersed or low poly-dispersity particles) ${ }^{19}$. Moreover, dispersed lipid particles have great potential for engaging in targeted and tracked delivery applications ${ }^{21-29}$. Upon dispersion, the cubic phase is retained within the lipid particles hence the term 'cubosomes' (Figure 1e) is used to describe them ${ }^{17}$. As compared to vesicles or liposomes, the cubosomes exhibit higher bilayer area to particle volume ratios ${ }^{19}$, in other words, the cubosomes possess much larger hydrophobic volume fractions (corresponding values of $0.59 \mathrm{~nm}^{3}$ as compared to $0.18 \mathrm{~nm}^{3}$ for $100 \mathrm{~nm}$ sized particles) ${ }^{21}$. This feature is very important for enhanced drug carrier capacity, especially of poorly water-soluble drugs ${ }^{19}$. In addition, the cubosomes are much more robust and stable owing to rather high viscous resistance to rupture ${ }^{19}$. 
There are several reports demonstrating the delivery applications of bulk lipid cubic phase ${ }^{30-39}$ as well as dispersed nanoparticles like cubosomes ${ }^{7,19,40-46}$ but there is hardly any record where both of the lipid systems are studied together under comparable conditions. In this work, we examined the release properties of bulk (non-dispersed) lipid cubic phase and it's dispersed form i.e. cubosomes for a model drug called aspirin (Figure 1a). Aspirin is an important drug exhibiting analgesic, antipyretic and anti-inflammatory properties. Moreover, it was shown to increase the solubility of cholesterol plaques within the membrane ${ }^{47}$ and increase its fluidity. However, aspirin has some predominant side effects including the toxicity of the gastrointestinal (GI) tract and rapid conversion into less desired products ${ }^{48}$. Therefore, its encapsulation into optimal delivery system would be largely beneficial ${ }^{48}$. We monitored the release of aspirin from bulk lipid cubic phase and cubosomes, in water and PBS media (Figure 1f), using UV-Vis spectroscopic technique.

\section{Materials and Methods}

\section{Materials:}

A lipid, Dimodan U/J ${ }^{\circledR}$ (DU) was generously provided by Danisco, A/S (Brabrand, Denmark). It is a distilled glyceride comprising 96\% monoglycerides (Figure 1a) and the rest are diglycerides and free fatty acids. Two major monoglyceride components in DU are linoleate (62\%) and oleate (25\%). Hence the hydrophobic part of DU mainly contains $\mathrm{C}_{18}$ chains (91\%). The following chemicals/items were purchased from Sigma-Aldrich (Dorset, UK): triblock copolymer Pluronic ${ }^{\circledR}$ F127 (PEO99-PPO $67-\mathrm{PEO}_{99}$ ) (Figure 1a); used to stabilize lipid particlescubosomes, aspirin (Chemical name Acetylsalicylic acid); it is a salicylate drug (Figure 1a) used mainly as analgesic and antipyretic agent, phosphate buffer saline (PBS) tablets, and dialysis membrane sacks (with an average flat width of $35 \mathrm{~mm}$, MWCO 12,000 Da). All the chemicals were used as received without any further purification. DU was stored below $4^{\circ} \mathrm{C}$ whereas the other materials were kept at room temperature when not in use. Water used during the entire study was purified using Barnstead Nanopure, Thermoscientific (USA).

\section{Standard calibration curves of a drug in water and PBS}

$50 \mathrm{mg}$ of aspirin was dissolved separately in $10 \mathrm{ml}$ distilled deionised water (now on referred as 'water') and $10 \mathrm{ml} 0.01 \mathrm{M}$ PBS buffer (one tablet dissolved in $200 \mathrm{ml}$ of deionized water yields $0.01 \mathrm{M}$ phosphate buffer with $\mathrm{pH}$ 7.4, now on referred as ' $\mathrm{PBS}$ ') in $100 \mathrm{ml}$ volumetric 
flasks. The mixtures were sonicated for 5 min (Sonics \& Materials Vibra-Cell VCX750, Jencons, UK) to ensure the drug dissolution. Finally, the corresponding volumes were made up to the mark with water and PBS to make $0.5 \mathrm{mg} / \mathrm{ml}$ stock solutions. Standard solutions were prepared by appropriate dilutions of above stock solutions. The absorption spectra of aspirin solutions were determined in the ultra-violet visible (UV) range of 200-800 $\mathrm{nm}$ with the characteristic $\lambda_{\max }$ at $276 \mathrm{~nm}$ (UV-1600PC, VWR, UK). At least 10 dilutions, in the range of 0.1 to $100 \mu \mathrm{g} / \mathrm{ml}$ concentrations, were utilized to establish standard calibration curves at the $\lambda_{\max }$ (see supporting information Figure S1). All measurements were performed in triplicates at room temperature $\left(\sim 25^{\circ} \mathrm{C}\right)$.

\section{Preparation and loading of a drug in the cubic phase (bulk lipid phase)}

The bulk lipid phase was prepared by melting $2 \mathrm{~g}$ lipid (DU) in a $20 \mathrm{ml}$ glass vial followed by addition of equal amount of water (by weight) yielding a known Pn3m cubic phase ${ }^{49}$ (Figure 1c). Drug-loaded bulk phase was prepared as follows: a mixture of appropriate drug concentration and $1 \mathrm{~g}$ molten DU was stirred (using magnetic stirrer bar) for $30 \mathrm{~min}$ at $50{ }^{\circ} \mathrm{C}$ followed by an addition of $1 \mathrm{~g}$ water. During the release experiments, further $9 \mathrm{~g}$ water was added to $1 \mathrm{~g}$ cubic phase in order to maintain comparable concentrations with cubosomes solution. Hydrated phase was physically mixed with the spatula and allowed to stand overnight at room temperature.

\section{Preparation and loading of a drug in cubosomes (dispersed lipid particles)}

The nanostructured lipid particle dispersions i.e. cubosome solutions, were prepared according to the published method albeit slightly different parameters and compositions ${ }^{50} .500 \mathrm{mg}$ of molten lipid (DU) was added to the $20 \mathrm{ml}$ glass vial and topped with $9.5 \mathrm{~g}$ F127 stabilizer solution (already prepared by dissolving 0.5\% Pluronic F127 powder in $100 \mathrm{~g}$ water). The resulting (10 g) mixture was ultra-sonicated (Sonics \& Materials Vibra-Cell VCX750, Jencons, UK) for 5 min with a continuous pulse using 35\% of the maximum power. Sample (milky fluid emulsion, Figure 1e) became hot due to ultra-sonication and was left to cool down to room temperature before further usage. Drug-loaded samples were prepared by stirring appropriate weight of a drug in above cubosome solution for about $20 \mathrm{~min}$ at $50{ }^{\circ} \mathrm{C}$.

\section{Drug lipid mixtures and a release set-up}

$10 \mathrm{~g}$ of drug-loaded bulk cubic phase mixture (containing $1 \mathrm{~g}$ lipid cubic phase and $9 \mathrm{~g}$ water) and $10 \mathrm{~g}$ dispersed cubosomes were loaded with a range of drug concentrations to obtain final samples of $2 \%, 4 \%, 6 \%$ and $10 \% \mathrm{wt} / \mathrm{wt}$ drug/lipid. Above samples were carefully transferred 
into dialysis membrane sacs (which were prepared by heating in pure water for $10 \mathrm{~min}$ at 80 $\left.{ }^{\circ} \mathrm{C}\right)$. Both ends of membrane sacs were tightly tied to avoid sample leakage. Finally the membrane sacs were individually immersed into $200 \mathrm{ml}$ of either water or PBS in $500 \mathrm{ml}$ beakers. $3 \mathrm{ml}$ solution from aforementioned release set-up (beaker) was collected after periodic timescales including $t=0 \mathrm{~min}$, and the UV-Vis spectroscopic data was measured at $\lambda_{\max }$ of 276 $\mathrm{nm}$. After measurements, the solutions were poured back into the original reservoirs to maintain accumulative release conditions. Studied drug concentrations were well within the sink conditions ${ }^{51}$.

\section{Entrapment efficiency (\%) and drug loading (\%) in cubosomes and bulk cubic phase}

$1 \mathrm{gm}$ of drug (2\%) loaded dispersed cubosomes were added to $1.5 \mathrm{ml}$ Eppendorf tube and centrifuged at 13,200 rpm for 10 min (Spectrafuge 24D from Jencons Pls). The fluid phase was transferred into an empty Eppendorf tube and centrifuged for further $10 \mathrm{~min}$. Majority of the cubosome particles were removed by this method; however, the emulsion retained the opacity. The remaining lipid was separated by using Amicon Ultra-0.5 Centrifugal Filter Unit with Ultracel-3 membranes (NMWL 3 KDa, Millipore, USA) with 20 min centrifugation at 13,200 rpm. The separated aqueous phase was diluted with water and the absorbance at $276 \mathrm{~nm}$ was measured using UV-Vis spectroscopic technique. The entrapment efficiency (EE) and drug loading (DL) was determined using following formulae ${ }^{52}$.

$$
\begin{aligned}
& E E=\left(1-\frac{C_{U}}{C_{T}}\right) \times 100 \\
& D L=\left(\frac{C_{T}-C_{U}}{C_{L}}\right) \times 100 .
\end{aligned}
$$

where, $C_{U}$ is the concentration of non-entrapped drug (free unloaded drug), $C_{T}$ is the concentration of drug added to the cubosome dispersion and $C_{L}$ is the total lipid content. The EE and DL for other cubosome dispersions (with 4, 6, and 10\% drug) were determined in the same manner as descried above. The excess water (aqueous phase) from drug-loaded bulk cubic phase system was separated simply by decanting into empty cuvettes followed by recording their absorbance at $276 \mathrm{~nm}$. The EE and DL values for 2, 4, 6 and 10\% drug-loaded bulk cubic phase samples were determined subsequently using above formulae (eqs. 1 and 2).

\section{Particle size analysis of cubosome dispersions}

The mean particle size and size distribution of cubosome dispersions were measured using Zetasizer Nano ZS instrument (Malvern Instruments, UK) operated at $25^{\circ} \mathrm{C}$. 


\section{Detecting the type of lipid nanostructure using Small angle $X$-ray scattering}

Small angle X-ray scattering (SAXS) was used to detect the type of lipid nanostructure of bulk and dispersed lipid systems (Figure 2). The SAXSpace instrument (Anton Paar, Graz, Austria) at University of Leeds was employed for this purpose. The details of the instrument and measurement are published earlier ${ }^{53}$. The instrument is based on $\mathrm{Cu}$-anode operating at $40 \mathrm{kV}$ and $50 \mathrm{~mA}$. Samples were measured using capillary sample holder controlled at $25 \pm 0.1^{\circ} \mathrm{C}$. Typical exposure times of $300 \mathrm{sec}$ were sufficient to obtain patterns with well-resolved peaks (Figure 2).

\section{Results and Discussion}

Nanostructural characterization and drug loading capacity of bulk cubic phase and cubosomes

The type of nanostructural self-assembly was determined using SAXS for drug-loaded and native lipid systems. The bulk DU formed Pn3m type bicontinuous cubic phase in excess water ${ }^{49}$, which was retained upon addition of $10 \%$ drug (Figure 2a). In case of dispersed lipid systems, the addition of stabilizer molecules usually cause phase transition into $\operatorname{Im} 3 m$ type bicontinuous cubic phase ${ }^{20,54-55}$ (Figure 2b).
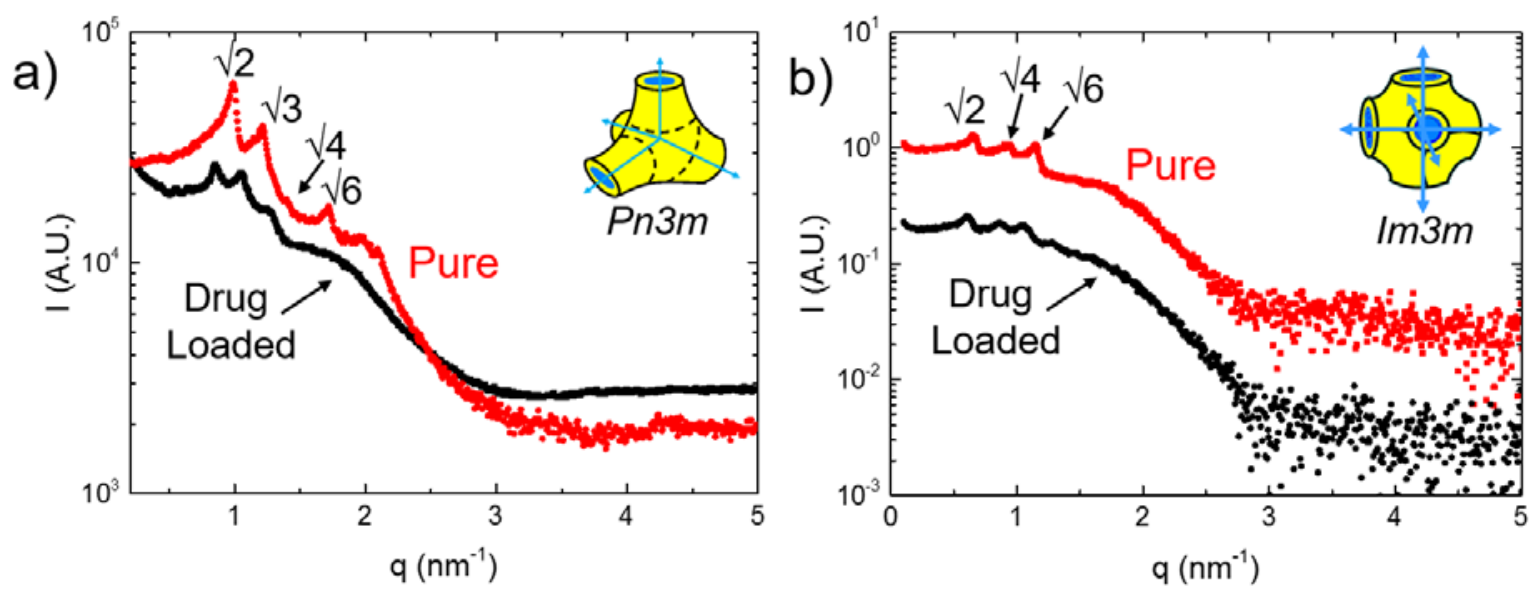

Figure 2: Detection of the type of lipid self-assembly using SAXS: a) Pn3m cubic phase was observed in pure and drug-loaded (10\% drug) bulk lipid system. b) Im3m cubic phase was observed in pure and drug-loaded (10\% drug) dispersed cubosome system. Characteristic Bragg's diffractions for $\operatorname{Pn} 3 m(\sqrt{2}, \sqrt{3}, \sqrt{ } 4, \sqrt{6})$ cubic phase and $\operatorname{Im} 3 m(\sqrt{2}, \sqrt{4}, \sqrt{6})$ cubic phase are shown near corresponding peaks. Aqueous channels are indicated by arrows. 
The ultrasonic dispersion process created discrete lipid particles with an internal nanostructure of cubic phase. These cubosomes exhibited sub-micron size as verified by the particle size analysis (for plot see supporting information Figure S2). The particle size values for native and drug-loaded cubosomes are listed in Table 1.

\begin{tabular}{|c|c|c|}
\hline $\begin{array}{c}\text { Drug in Dispersion } \\
\text { (Wt\% per Wt of lipid) }\end{array}$ & $\begin{array}{c}\text { Average Particle Size } \\
\mathbf{n m}\end{array}$ & $\begin{array}{c}\text { PDI } \\
\text { (Poly dispersity index) }\end{array}$ \\
\hline 0 & $194 \pm 4$ & 0.210 \\
\hline 2 & $184 \pm 3$ & 0.192 \\
\hline 4 & $183 \pm 3$ & 0.207 \\
\hline 6 & $182 \pm 2$ & 0.205 \\
\hline 10 & $184 \pm 1$ & 0.184 \\
\hline
\end{tabular}

Table 1. Particle size and polydispersity indices of native and drug-loaded cubosomes measured by dynamic light scattering technique Zetasizer Nano ZS.

An entrapment efficiency of bulk lipid cubic phase in excess water and cubosomes dispersions was calculated using eq. 1 as shown in Table 2. Similarly, drug loading in lipid cubic phase and cubosome particles was calculated using eq 2 and shown in Table 2. The EE and DL values for bulk cubic phase were higher as compared cubosome system. The low DL values demonstrate that the drug is preferably located in the aqueous phase rather than lipid structures. Upon encapsulation in lipid systems and subsequent dehydration, the structural features of a drug (aspirin) are generally protected as reported in our previous work ${ }^{56}$. However, lipid systems employed in the current study were always in excess water condition permitting the drug encapsulation in both, lipid and aqueous regions (Table 2).

\begin{tabular}{|c|c|c|c|c|}
\hline $\begin{array}{c}\text { Drug } \\
\text { loading }\end{array}$ & $\begin{array}{c}\text { EE bulk cubic phase } \\
\text { in excess water }\end{array}$ & $\begin{array}{c}\text { EE cubosome } \\
\text { dispersion }\end{array}$ & $\begin{array}{c}\text { DL } \\
\text { cubic phase }\end{array}$ & $\begin{array}{c}\text { DL } \\
\text { cubosomes }\end{array}$ \\
\hline $\begin{array}{c}\text { Wt \%/Wt of } \\
\text { lipid }\end{array}$ & \% & \% & \% & \% \\
\hline 2 & 84.1 & 61.9 & 0.39 & 0.25 \\
\hline 4 & 98.7 & 67.6 & 0.91 & 0.54 \\
\hline 6 & 98.5 & 71.3 & 1.36 & 0.86 \\
\hline 10 & 96.6 & 71.6 & 2.22 & 1.43 \\
\hline
\end{tabular}

Table 2. Entrapment efficiency (EE) and Drug Loading (DL) in bulk lipid phase and dispersed cubosomes. 


\section{Drug release from bulk cubic phase}

Referring to the standard calibration curves obtained by dissolving various concentrations of a drug in water and PBS (supporting information Figure S1), the measured absorbance values at $\lambda_{\max }$ of $276 \mathrm{~nm}$ were translated into percentage (\%) release values. These values were then plotted against time recorded in hours for release in water (Figure 3) and in PBS (Figure 4) for bulk cubic phase.
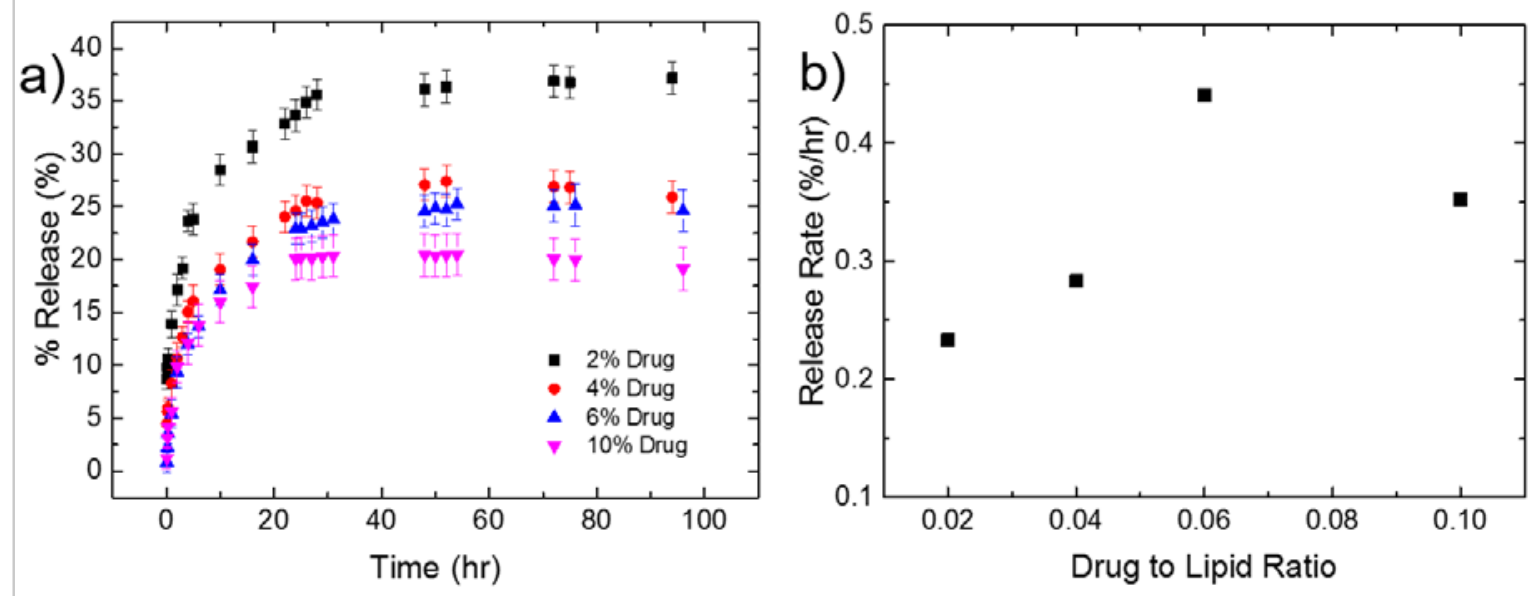

Figure 3: Drug release from bulk cubic phase in water: a) Percent (\%) drug release calculated from corresponding absorbance values (at $\lambda_{\max }$ of $276 \mathrm{~nm}$ ) are shown against time in hours for 2, 4, 6 and 10\% drug. b) Curves in a) were fitted with Korsmeyer-Peppas equation to obtain the release rates $(\% / h r)$, which are plotted against the drug to lipid ratio values.

The curves in Figures 3 and 4 essentially followed typical drug release kinetics. Their logarithmic plots fitted well (with correlation coefficients- $\mathrm{R}^{2}$ values $>0.96$ ) with characteristic Korsmeyer-Peppas equation (eq. 3) ${ }^{57-58}$; for representative fits, see supporting information Figure S3.

$M_{t} /_{M_{\infty}}=K t^{n}$

where, $M_{t}$ is the amount of drug released at time ' $t$ ', $M_{\infty}$ is the total amount of drug in the formulation, $K$ is a kinetic constant, $n$ is the exponent - characteristic of the release mechanism and $t$ is the release time in hours.

The drug release in water medium became insignificant after about 30 hrs (Figure 3a) but it was gradual and well-detectable up to about studied 96 hrs in case of PBS release medium 
(Figure 4a). Slopes of the fit lines, i.e. release rates in \%/hr follows a linear trend except for 10\% drug sample (Figures $3 \mathrm{~b}$ and $4 \mathrm{~b}$ ). The corresponding slopes of these lines were comparable: $0.05198 \% \mathrm{Wt} \% \mathrm{hr}^{-1}$ for release in water (Figure 3b) and $0.04701 \% \mathrm{Wt} \% \mathrm{hr}^{-1}$ for release in PBS medium (Figure 4b). Both release rates are dependent on the drug concentration and follow first-order kinetics.
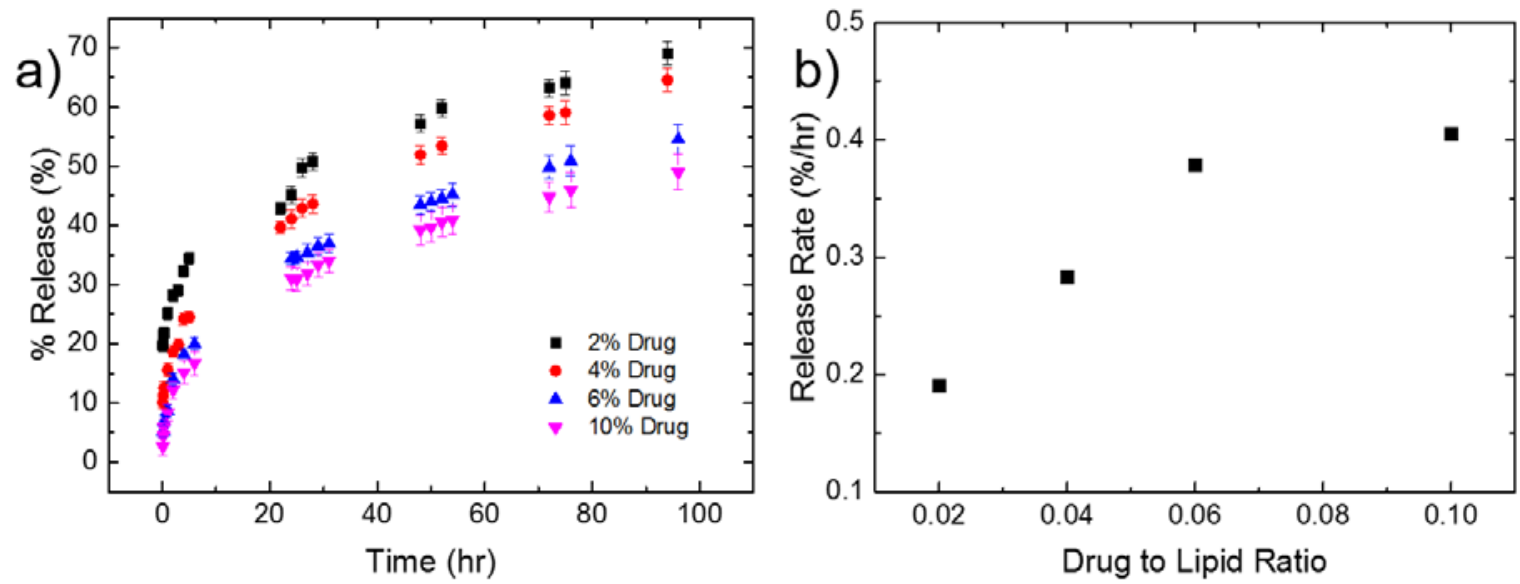

Figure 4: Drug release from bulk cubic phase in PBS: a) Percent (\%) drug release calculated from corresponding absorbance values (at $\lambda_{\max }$ of $276 \mathrm{~nm}$ ) are shown against time in hours for 2, 4, 6 and 10\% drug. b) Curves in a) were fitted with Korsmeyer-Peppas equation to obtain the release rates $(\% / \mathrm{hr})$, which are plotted against the drug to lipid ratio values.

\section{Drug Release from dispersed cubosomes}

The release trends from cubosomes (Figure 5) virtually followed similar release patterns as from bulk cubic phase (Figures 3 and 4). The release in water saturated after about 30 hrs whereas it continued gradually in case of PBS. 

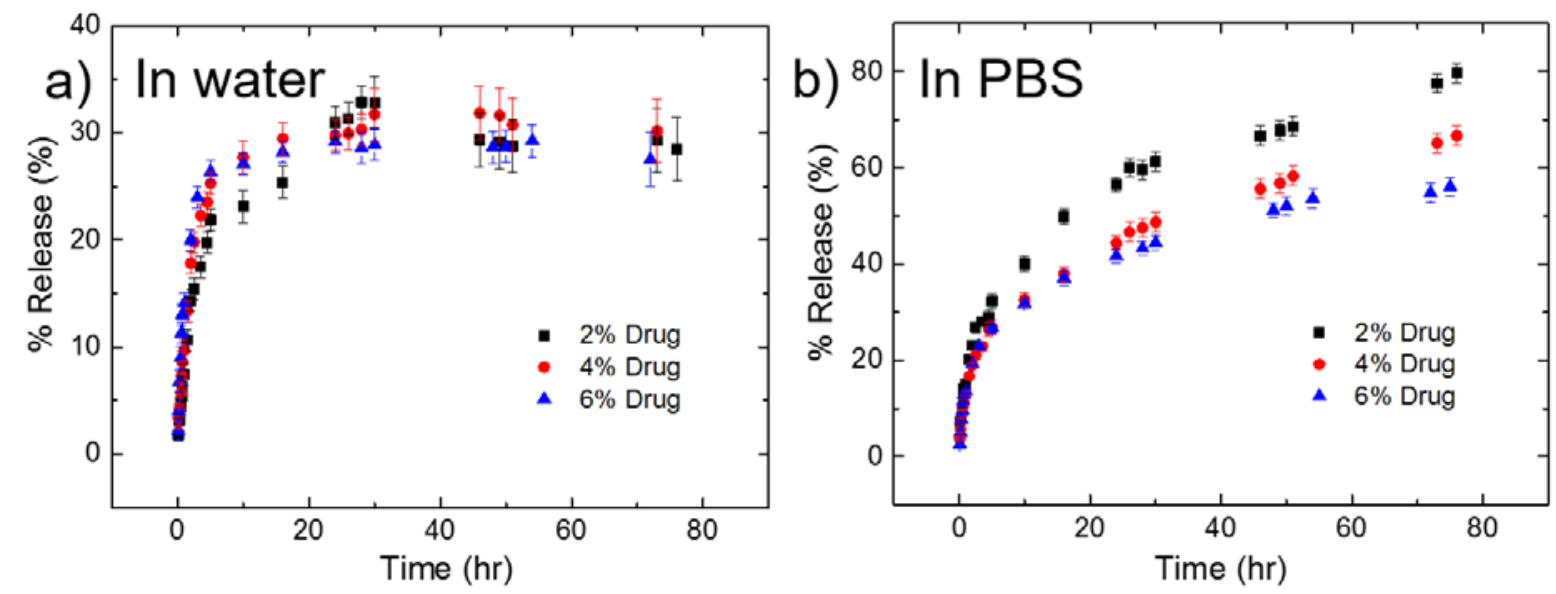

Figure 5: Drug release from dispersed cubosomes in a) water and b) PBS: Percent (\%) drug release calculated from corresponding absorbance values (at $\lambda_{\max }$ of $276 \mathrm{~nm}$ ) is plotted against time in hours for 2, 4, 6 and 10\% drug/lipid for release in water a) and in PBS b).

However, the release from cubosomes in water (Figure 5a) appears to be less dependent on the drug concentration as evident from the closely positioned release curves. Nevertheless, the release curves on log-log scale, both in water and in PBS, were fitted using Korsmeyer-Peppas equation (eq. 3$)^{57}$ with typical $\mathrm{R}^{2}$ values $>0.93$.

\section{Sustained drug release from bulk cubic phase compared to cubosomes}

Comparing the release rates from cubic phases and cubosomes (Figure 6), it is clear that the release from cubosomes was faster than the release from bulk cubic phases. The release of $2 \%$ drug from bulk cubic phase was found to be three-folds $(0.233 \% / \mathrm{hr})$ sustained than the release from cubosomes $(0.658 \% / \mathrm{hr})$ when measured in water; whereas it was twice more sustained from bulk cubic phase $(0.190 \% / \mathrm{hr})$ than from cubosomes $(0.402 \% / \mathrm{hr})$ in PBS. The same trend was also observed for the release rate values of $4 \%$ drug in water $(0.283 \% / h r$ for bulk cubic phase and $0.637 \% / \mathrm{hr}$ for cubosomes) and in PBS (0.282 \%/hr for bulk cubic phase and 0.407 $\% / \mathrm{hr}$ for cubosomes). The releases for $6 \%$ and $10 \%$ drug from cubosomes were also faster than the releases from bulk cubic phases, both in water and PBS release media. 

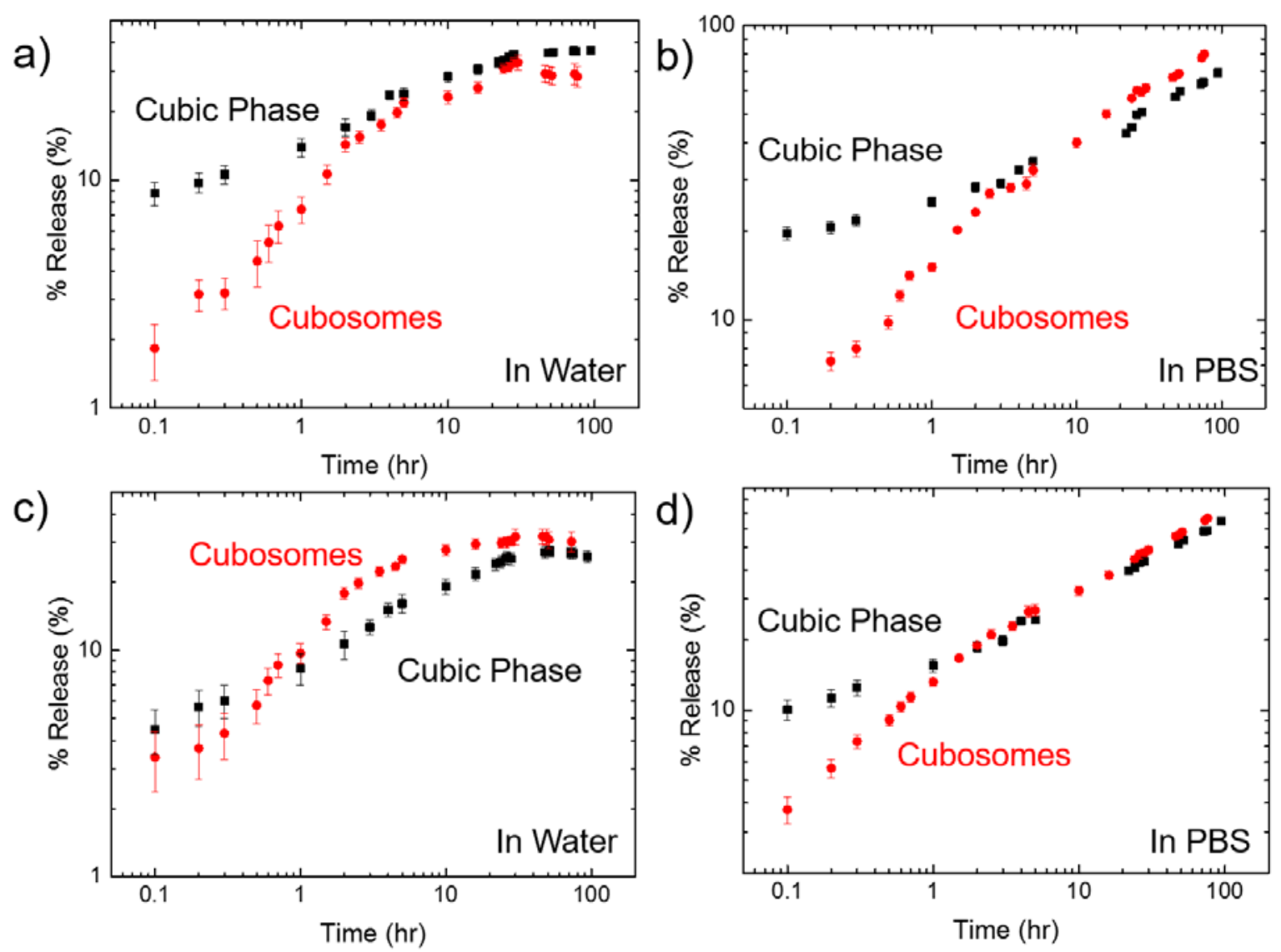

Figure 6: Comparison of drug release from bulk cubic phase and cubosomes: \% release for $2 \%$ drug in a) water and b) PBS and $4 \%$ drug in c) water and d) PBS are shown with time in hrs.

Fittingness of Korsmeyer-Peppas equation to almost all curves in both lipid systems helps to understand the underlying release mechanisms. Analysis of the exponent $n$ (eq. 3) suggest that the release mechanism is a Fickian diffusion when $n$ value is less than or equal to $0.45(n \leq 0.45)$. The release from bulk cubic phase, both in water and in PBS followed this mechanism. Apparent Fickian diffusion is known to contribute in sustaining the release of small molecules from bulk cubic phases ${ }^{30-31,35-39}$. This can be easily attributed to the highly tortuous porous morphology of cubic phases. In order to release from the bulk cubic phase, the drug molecule has to navigate through the lengthy aqueous network assimilated by long and continuous lipid bilayer architecture. On the contrary, the release from cubosomes is rapid and less sustained (Figure 6). This is due to smaller $(<200 \mathrm{~nm} \text {, Table } 1)^{53}$ particle size correlated to smaller length scales for the drug transport, and too high surface area accountable to the burst release kinetics $^{59}$. The release from cubosomes in water displayed $n$ value below 0.45 but for the 
release in PBS the $n$ value was greater than $0.45(n=0.63)$ depicting anomalous or non-Fickian diffusion kinetics ${ }^{57-58}$.

A few recent reports demonstrate that the release rates from lipid systems can be controlled by fine-tuning nanostructural type and dimensions of the self-assembled phases ${ }^{30-32,60-62}$. For instance, if the nanostructure is hexagonal phase, the release can be more controlled in comparison to the open structured cubic nanostructures ${ }^{34,63}$. Moreover, the release could be administered via stimuli responsive ( $\mathrm{pH}$, light or temperature induced) triggers ${ }^{63-65}$. We have recently demonstrated that it is possible to sustain the release from cubosomes by encapsulating them into hydrogel films ${ }^{56,66}$. The release, in this case, is believed to occur in multiple steps involving hydration of the hydrogel film, swelling and subsequent erosion of the hydrogel, opening of the aqueous channels of cubosomes, burst release and finally the dissolution of the hydrogel matrix ${ }^{56}$.

The nanoscale design and dimensions of the cubic self-assemblies further influence the release kinetics as follows. The lipid used in this work forms Pn3m type cubic phase in pure water ${ }^{49}$ (Figure 2a). This phase exhibits four aqueous channels (meeting at tetrahedral angle) whereas the internal self-assembly of cubosomes is found to be $\operatorname{Im} 3 m$ phase (Figure 2b) consisting six aqueous channels (meeting at right angles). Moreover, the aqueous channels of $\operatorname{Im} 3 m$ phase are usually larger than Pn3m phase for the same lipid system, see Table 3. Largely hydrophilic block co-polymer stabilizers, similar to F127 used in this work, are known to alter the average molecular shapes of cubic phase forming lipids ${ }^{20,54}$; most common result is the formation of Im3m phase in the internal cores of dispersed cubosomes ${ }^{55}$.

\begin{tabular}{|c|c|c|c|c|c|}
\hline $\begin{array}{c}\text { Physical } \\
\text { form of lipid } \\
\text { system }\end{array}$ & $\begin{array}{c}\text { Concentration } \\
\text { of drug Wt\% } \\
\text { per lipid Wt. }\end{array}$ & $\begin{array}{c}\text { Type of self- } \\
\text { assembled } \\
\text { cubic phase }\end{array}$ & $\begin{array}{c}\text { No. of } \\
\text { aqueous } \\
\text { channels }\end{array}$ & $\begin{array}{c}\text { Lattice } \\
\text { Parameter } \\
(\boldsymbol{a}), \boldsymbol{\AA}\end{array}$ & $\begin{array}{c}\text { Diameter of } \\
\text { aqueous } \\
\text { channel (dw), } \mathbf{\AA}\end{array}$ \\
\hline $\begin{array}{c}\text { Bulk cubic } \\
\text { phase }\end{array}$ & 0 & $P n 3 m$ & 4 & 89.5 & 37.9 \\
\hline $\begin{array}{c}\text { Bulk cubic } \\
\text { phase }\end{array}$ & 10 & $P n 3 m$ & 4 & 103.2 & 48.7 \\
\hline $\begin{array}{c}\text { Dispersed } \\
\text { cubosomes }\end{array}$ & 0 & $\operatorname{Im} 3 m$ & 6 & 135.6 & 50.9 \\
\hline $\begin{array}{c}\text { Dispersed } \\
\text { cubosomes }\end{array}$ & 10 & $\operatorname{Im} 3 m$ & 6 & 146.7 & 57.8 \\
\hline
\end{tabular}


Table 3. Nanoscale dimensions of lipid cubic phases formed in bulk system and dispersed systems, before and after drug loading. Lattice parameters and diameters of aqueous channels were calculated according to the formulae listed in reference ${ }^{67}$.

Encapsulation of a drug further elevates diameters of aqueous channels by about $22 \%$ in bulk cubic phase while 12\% in cubosomes (Table 3). Thus, the number of aqueous channels and their sizes are greater in the cubic phase $(\mathrm{Im} 3 \mathrm{~m})$ of cubosomes as compared to the bulk cubic phase (Pn3m), which theoretically contribute to rather rapid release from cubosomes.

\section{Efficient release in PBS than in water}

The drug release from all samples, in bulk cubic phases and cubosomes, in PBS was far more efficient (more than double in some cases) than the release in water (Figure 7, Table 4). PBS medium ( $\mathrm{pH} 7.4$ ) is generally referred to be the physiological condition where drug release kinetics is usually different than in ambient and pure conditions.
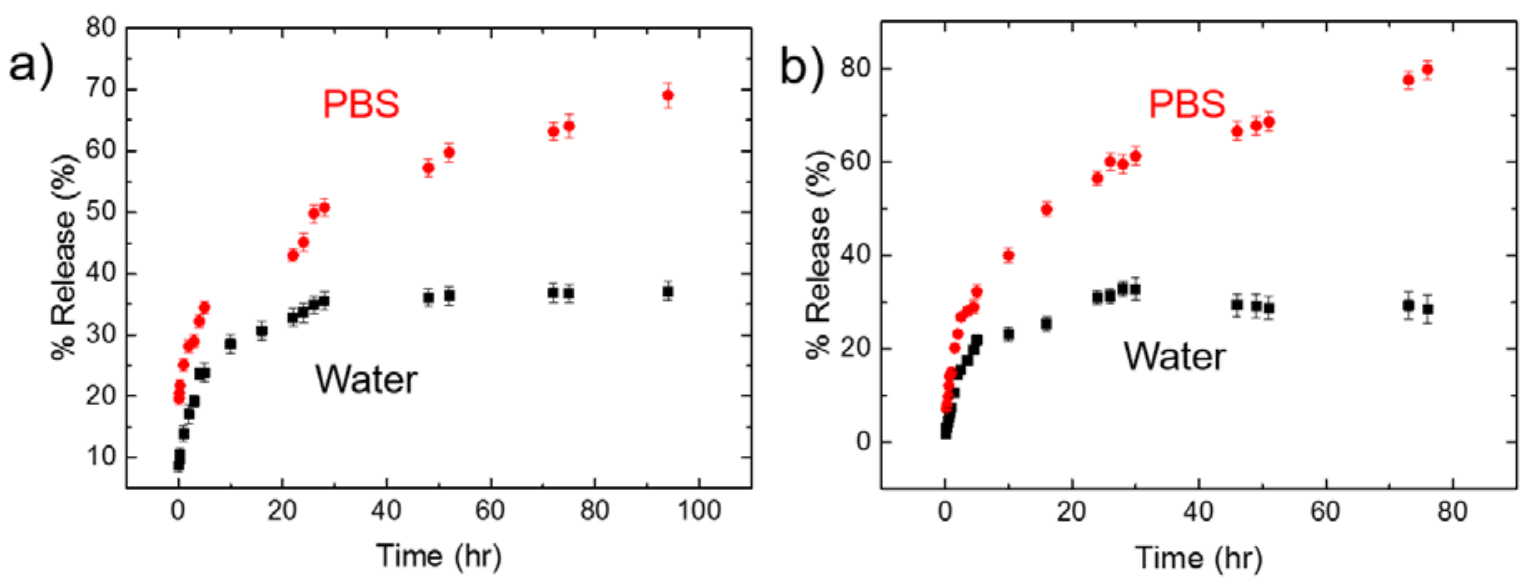

Figure 7: Drug release in PBS compared to water: a) Percent (\%) drug release in water (solid squares) and in PBS (solid circles) for 2\% drug/lipid is shown for a) bulk cubic phase and b) cubosome system. Note that the representative data plots for $2 \%$ drug/lipid samples are shown here; for remaining data plots (4\%, 6\% and 10\% drug/lipid) see supporting information Figure S4. 


\begin{tabular}{|c|c|c|c|c|}
\hline $\begin{array}{c}\text { Drug (Wt\% / } \\
\text { Wt of lipid) }\end{array}$ & $\begin{array}{c}\text { Bulk Cubic Phase } \\
\text { in Water, \% }\end{array}$ & $\begin{array}{c}\text { Bulk Cubic } \\
\text { Phase in PBS, \% }\end{array}$ & $\begin{array}{c}\text { Cubosomes in } \\
\text { Water, \% }\end{array}$ & $\begin{array}{c}\text { Cubosomes in } \\
\text { PBS, \% }\end{array}$ \\
\hline 2 & 33.65 & 45.12 & 30.93 & 56.53 \\
\hline 4 & 24.62 & 41.11 & 29.47 & 44.39 \\
\hline 6 & 22.91 & 34.39 & 29.16 & 41.67 \\
\hline 10 & 20.09 & 31.05 & 18.23 & 38.96 \\
\hline
\end{tabular}

Table 4. The \% release at 24 hrs from both lipid systems in water and in PBS.

Main advantages of employing PBS for release studies include the retention of constant $\mathrm{pH}$, prevention from denaturation and/or conformational changes, resembling the bodily conditions (isotonic) and nontoxicity to cells ${ }^{68}$. However, biological buffers, and also associated $\mathrm{pH}$ affects the membrane properties ${ }^{68-69}$. It was envisaged that the buffer molecules interact with hydrophilic lipid head groups and intercalate within the head group region facilitating the reduction of membrane bending modulus and increase in number of membrane fluctuations ${ }^{69}$. The overall effect of these events is revealed in the form of swelling ${ }^{68}$ and/or softening of lipid membranes ${ }^{69}$. This could be directly linked with the continued and efficient release from lipid carriers in PBS medium in contrast to the low and ceased release in pure water medium (Figure 7, Table 4).

The release (\%) measured after 24 hrs for bulk cubic phase and cubosomes in both medium, i.e. water and PBS always decreased with the increase in drug: lipid ratio (Table 4). This again confirms that the release rate depends on the drug concentration, thus following the first-order release kinetics.

\section{Conclusions and perspectives}

In this work we have compared the release properties of two different lipid nanocarrier systems. Bulk lipid cubic phase and cubosomes were studied under similar conditions of drug to lipid ratios and the volumes of release media. The release in both lipid systems followed a mechanism described by Korsmeyer-Peppas equation revealing the Fickian diffusion through the porous matrix ${ }^{57-58}$. However, the diffusion from cubosomes in PBS medium was anomalous, involving either or a combination of non-Fickian diffusion and erosion $^{57-58}$. The latter may correspond to the interaction of PBS buffer molecules with lipid bilayer structures ${ }^{68-69}$ that are enclosed by small cubosome particles. This can be 
verified from the efficient and rapid release in PBS medium compared to the almost ceased release in water medium (Figure 7).

The release from bulk lipid systems was sustained with respect to the dispersed colloidal cubosomes due to apparent reasons. Although both lipid systems exhibit self-assembled cubic nanostructures with highly tortuous porous networks, the effective length scales experienced by drug molecules differ enormously. Cubosomes particles are small in size with rather high interfacial area leading to the burst release while longer length scales of bulk cubic phase enable prolonged drug release. Moreover, the wider and more number of aqueous channels of $\operatorname{Im} 3 \mathrm{~m}$ cubic phase contribute in the enhanced release from cubosomes. At this stage we cannot comment on the role of overall high viscosity of bulk cubic phases in the release kinetics and leave it for further in-depth studies. This study shades light on important aspect of comparing two emerging drug delivery systems based on the nanoscale self-assembly of lipid molecules in aqueous medium. The results obtained are potentially useful for predictable design and optimization of these nanocarrier systems.

\section{Acknowledgements}

We would like to thank Prof Michael Rappolt and Dr Amin Sadeghpour from university of Leeds for their support with the SAXS instrument. We also thank Mark Higham for supporting with additional experiments.

\section{Reference}

1. Kulkarni, C. V., Lipid crystallization: from self-assembly to hierarchical and biological ordering. Nanoscale 2012, 4 (19), 5779-5791.

2. Seddon, J. M.; Templer, R. H., Polymorphism of lipid-water systems. In Handbook of Biological Physics, Lipowsky, R.; Sackmann, E., Eds. Elsevier Science B.V. Amsterdam: 1995; Vol. 1, pp 97-160.

3. Seddon, J. M.; Templer, R. H., Cubic Phases of Self-assembled Amphiphilic Aggregates. Philosophical transactions. Mathematical, physical, and engineering sciences 1993, 344 (1672), 377-401.

4. Scriven, L. E., Equilibrium bicontinuous structure. Nature 1976, 263 (5573), 123-125.

5. Eriksson, P. O.; Lindblom, G., Lipid and water diffusion in bicontinuous cubic phases measured by NMR. Biophysical Journal 1993, 64 (1), 129-136.

6. Seddon, J. M.; Zeb, N.; Templer, R. H.; McElhaney, R. N.; Mannock, D. A., An Fd3m Lyotropic Cubic Phase in a Binary Glycolipid/Water System. Langmuir 1996, 12 (22), 5250-5253. 
7. Nazaruk, E.; Majkowska-Pilip, A.; Bilewicz, R., Lipidic Cubic-Phase NanoparticlesCubosomes for Efficient Drug Delivery to Cancer Cells. ChemPlusChem 2017, 82 (4), 570-575.

8. van 't Hag, L.; Gras, S. L.; Conn, C. E.; Drummond, C. J., Lyotropic liquid crystal engineering moving beyond binary compositional space - ordered nanostructured amphiphile self-assembly materials by design. Chemical Society reviews 2017.

9. Sadhale, Y.; Shah, J. C., Glyceryl monooleate cubic phase gel as chemical stability enhancer of cefazolin and cefuroxime. Pharm Dev Technol 1998, 3 (4), 549-56.

10. Kimber, R. G. E.; Walker, A. B.; Schroder-Turk, G. E.; Cleaver, D. J., Bicontinuous minimal surface nanostructures for polymer blend solar cells. Physical Chemistry Chemical Physics 2010, 12 (4), 844-851.

11. Tomas, L., From entangled membranes to eclectic morphologies: cubic membranes as subcellular space organizers. FEBS Letters 1995, 369 (1), 13-17.

12. Anderson, D. M.; Gruner, S. M.; Leibler, S., Geometrical aspects of the frustration in the cubic phases of lyotropic liquid crystals. PNAS 1988, 85 (15), 5364-5368.

13. Gradzielski, M.; Hoffmann, H.; Panitz, J.-C.; Wokaun, A., Investigations on L2 Phase and Cubic Phase in the System AOT/1 -Octanol/Water. Journal of Colloid and Interface Science 1995, 169 (1), 103-118.

14. Cheng, A.; Hummel, B.; Qiu, H.; Caffrey, M., A simple mechanical mixer for small viscous lipid-containing samples. Chemistry and Physics of Lipids 1998, 95 (1), 11-21.

15. Cherezov, V.; Caffrey, M., A simple and inexpensive nanoliter-volume dispenser for highly viscous materials used in membrane protein crystallization. Journal of Applied Crystallography 2005, 38 (2), 398-400.

16. Nollert, P., From test tube to plate: a simple procedure for the rapid preparation of microcrystallization experiments using the cubic phase method. Journal of Applied Crystallography 2002, 35, 637-640.

17. Gustafsson, J.; Ljusberg-Wahren, H.; Almgren, M.; Larsson, K., Cubic Lipid-Water Phase Dispersed into Submicron Particles. Langmuir 1996, 12 (20), 4611-4613.

18. Gustafsson, J.; Ljusberg-Wahren, H.; Almgren, M.; Larsson, K., Submicron Particles of Reversed Lipid Phases in Water Stabilized by a Nonionic Amphiphilic Polymer. Langmuir 1997, 13 (26), 6964-6971.

19. Garg, G.; Saraf, S.; Saraf, S., Cubosomes: An Overview. Biological \& pharmaceutical bulletin 2007, 30 (2), 350-353.

20. Kulkarni, C. V.; Glatter, O., Hierarchically Organized Systems Based on Liquid Crystalline Phases. In Self-Assembled Supramolecular Architectures: Lyotropic Liquid Crystals, Garti, N., Ed. John Wiley \& Sons, Inc.: 2012.

21. Meli, V.; Caltagirone, C.; Falchi, A. M.; Hyde, S. T.; Lippolis, V.; Monduzzi, M.; ObiolsRabasa, M.; Rosa, A.; Schmidt, J.; Talmon, Y.; Murgia, S., Docetaxel-Loaded Fluorescent Liquid-Crystalline Nanoparticles for Cancer Theranostics. Langmuir 2015, 31 (35), 956675.

22. Azhari, H.; Strauss, M.; Hook, S.; Boyd, B. J.; Rizwan, S. B., Stabilising cubosomes with Tween 80 as a step towards targeting lipid nanocarriers to the blood-brain barrier. European Journal of Pharmaceutics and Biopharmaceutics 2016, 104, 148-155.

23. Kadhum, W. R.; Oshizaka, T.; Ichiro, H.; Todo, H.; Sugibayashi, K., Usefulness of liquidcrystal oral formulations to enhance the bioavailability and skin tissue targeting of p-amino benzoic acid as a model compound. European Journal of Pharmaceutical Sciences 2016, 88, 282-290.

24. Miceli, V.; Meli, V.; Blanchard-Desce, M.; Bsaibess, T.; Pampalone, M.; Conaldi, P. G.; Caltagirone, C.; Obiols-Rabasa, M.; Schmidt, J.; Talmon, Y.; Casu, A.; Murgia, S., In vitro 
imaging of [small beta]-cells using fluorescent cubic bicontinuous liquid crystalline nanoparticles. RSC Advances 2016, 6 (67), 62119-62127.

25. Angelova, A.; Garamus, V. M.; Angelov, B.; Tian, Z.; Li, Y.; Zou, A., Advances in structural design of lipid-based nanoparticle carriers for delivery of macromolecular drugs, phytochemicals and anti-tumor agents. Advances in Colloid and Interface Science 2017.

26. Angelova, A.; Angelov, B., Dual and multi-drug delivery nanoparticles towards neuronal survival and synaptic repair. Neural regeneration research 2017, 12 (6), 886-889.

27. Angelova, A.; Angelov, B.; Mutafchieva, R.; Lesieur, S., Biocompatible Mesoporous and Soft Nanoarchitectures. Journal of Inorganic and Organometallic Polymers and Materials 2015, 25 (2), 214-232.

28. Angelov, B.; Angelova, A.; Drechsler, M.; Garamus, V. M.; Mutafchieva, R.; Lesieur, S., Identification of large channels in cationic PEGylated cubosome nanoparticles by synchrotron radiation SAXS and Cryo-TEM imaging. Soft Matter 2015, 11 (18), 36863692.

29. Angelova, A.; Angelov, B.; Drechsler, M.; Lesieur, S., Neurotrophin delivery using nanotechnology. Drug Discov Today 2013, 18 (23-24), 1263-71.

30. Meikle, T. G.; Yao, S.; Zabara, A.; Conn, C. E.; Drummond, C. J.; Separovic, F., Predicting the release profile of small molecules from within the ordered nanostructured lipidic bicontinuous cubic phase using translational diffusion coefficients determined by PFG-NMR. Nanoscale 2017, 9 (7), 2471-2478.

31. Nazaruk, E.; Miszta, P.; Filipek, S.; Górecka, E.; Landau, E. M.; Bilewicz, R., Lyotropic Cubic Phases for Drug Delivery: Diffusion and Sustained Release from the Mesophase Evaluated by Electrochemical Methods. Langmuir 2015, 31 (46), 12753-12761.

32. Negrini, R.; Sánchez-Ferrer, A.; Mezzenga, R., Influence of Electrostatic Interactions on the Release of Charged Molecules from Lipid Cubic Phases. Langmuir 2014, 30 (15), 4280-4288.

33. Nazaruk, E.; Szlęzak, M.; Górecka, E.; Bilewicz, R.; Osornio, Y. M.; Uebelhart, P.; Landau, E. M., Design and Assembly of pH-Sensitive Lipidic Cubic Phase Matrices for Drug Release. Langmuir 2014, 30 (5), 1383-1390.

34. Chen, Y.; Ma, P.; Gui, S., Cubic and Hexagonal Liquid Crystals as Drug Delivery Systems. BioMed Research International 2014, 2014, 12.

35. Rizwan, S. B.; Boyd, B. J.; Rades, T.; Hook, S., Bicontinuous cubic liquid crystals as sustained delivery systems for peptides and proteins. Expert Opin Drug Deliv 2010, 7 (10), 1133-44.

36. Nguyen, T. H.; Hanley, T.; Porter, C. J.; Larson, I.; Boyd, B. J., Phytantriol and glyceryl monooleate cubic liquid crystalline phases as sustained-release oral drug delivery systems for poorly water soluble drugs I. Phase behaviour in physiologically-relevant media. $J$ Pharm Pharmacol 2010, 62 (7), 844-55.

37. Lee, K. W.; Nguyen, T. H.; Hanley, T.; Boyd, B. J., Nanostructure of liquid crystalline matrix determines in vitro sustained release and in vivo oral absorption kinetics for hydrophilic model drugs. Int J Pharm 2009, 365 (1-2), 190-9.

38. Boyd, B. J.; Khoo, S. M.; Whittaker, D. V.; Davey, G.; Porter, C. J., A lipid-based liquid crystalline matrix that provides sustained release and enhanced oral bioavailability for a model poorly water soluble drug in rats. Int J Pharm 2007, 340 (1-2), 52-60.

39. Boyd, B. J.; Whittaker, D. V.; Khoo, S. M.; Davey, G., Lyotropic liquid crystalline phases formed from glycerate surfactants as sustained release drug delivery systems. Int J Pharm 2006, 309 (1-2), 218-26.

40. Avachat, A. M.; Parpani, S. S., Formulation and development of bicontinuous nanostructured liquid crystalline particles of efavirenz. Colloids and Surfaces B: Biointerfaces 2015, 126 (0), 87-97. 
41. Milošević, I.; Guillot, S.; Tadić, M.; Duttine, M.; Duguet, E.; Pierzchala, K.; Sienkiewicz, A.; Forró, L.; Saboungi, M.-L., Loading and release of internally self-assembled emulsions embedded in a magnetic hydrogel. Applied Physics Letters 2014, 104 (4), 043701.

42. Hinton, T. M.; Grusche, F.; Acharya, D.; Shukla, R.; Bansal, V.; Waddington, L. J.; Monaghan, P.; Muir, B. W., Bicontinuous cubic phase nanoparticle lipid chemistry affects toxicity in cultured cells. Toxicology Research 2014, 3 (1), 11-22.

43. Simovic, S.; Barnes, T. J.; Tan, A.; Prestidge, C. A., Assembling nanoparticle coatings to improve the drug delivery performance of lipid based colloids. Nanoscale 2012, 4 (4).

44. Chemelli, A.; Maurer, M.; Geier, R.; Glatter, O., Optimized Loading and Sustained Release of Hydrophilic Proteins from Internally Nanostructured Particles. Langmuir 2012, 28 (49), 16788-16797.

45. Nguyen, T.-H.; Hanley, T.; Porter, C. J. H.; Boyd, B. J., Nanostructured liquid crystalline particles provide long duration sustained-release effect for a poorly water soluble drug after oral administration. Journal of Controlled Release 2011, 153 (2), 180-186.

46. Zhao, X. Y.; Zhang, J.; Zheng, L. Q.; Li, D. H., Studies of cubosomes as a sustained drug delivery system. Journal of Dispersion Science and Technology 2004, 25 (6), 795-799.

47. Alsop, R. J.; Barrett, M. A.; Zheng, S.; Dies, H.; Rheinstadter, M. C., Acetylsalicylic acid (ASA) increases the solubility of cholesterol when incorporated in lipid membranes. Soft Matter 2014, 10 (24), 4275-86.

48. Singco, B.; Liu, L.-H.; Chen, Y.-T.; Shih, Y.-H.; Huang, H.-Y.; Lin, C.-H., Approaches to drug delivery: Confinement of aspirin in MIL-100(Fe) and aspirin in the de novo synthesis of metal-organic frameworks. Microporous and Mesoporous Materials 2016, 223, 254260.

49. Mezzenga, R.; Meyer, C.; Servais, C.; Romoscanu, A. I.; Sagalowicz, L.; Hayward, R. C., Shear Rheology of Lyotropic Liquid Crystals: A Case Study. Langmuir 2005, 21 (8), 3322.

50. Yaghmur, A.; de Campo, L.; Sagalowicz, L.; Leser, M. E.; Glatter, O., Emulsified Microemulsions and Oil-Containing Liquid Crystalline Phases. Langmuir 2005, 21 (2), 569-577.

51. O'Neil, M. J., The Merck Index - An Encyclopedia of Chemicals, Drugs, and Biologicals. Merck and Co., Inc.: Whitehouse Station, NJ, 2006.

52. Chen, Y.; Angelova, A.; Angelov, B.; Drechsler, M.; Garamus, V. M.; Willumeit-Romer, R.; Zou, A., Sterically stabilized spongosomes for multidrug delivery of anticancer nanomedicines. Journal of Materials Chemistry B 2015, 3 (39), 7734-7744.

53. Patil-Sen, Y.; Sadeghpour, A.; Rappolt, M.; Kulkarni, C. V., Facile Preparation of Internally Self-assembled Lipid Particles Stabilized by Carbon Nanotubes. Journal of Visualized Experiments 2016, (108), e53489.

54. Kulkarni, C. V.; Patil-Sen, Y.; Kulkarni, M.; Iglic, A., Biomolecules Altering the Lipid Molecular Shape in Model Non-Lamellar Membranes. Biophysical Journal 2015, 108 (2), 544a.

55. Guillot, S.; Moitzi, C.; Salentinig, S.; Sagalowicz, L.; Leser, M. E.; Glatter, O., Direct and indirect thermal transitions from hexosomes to emulsified micro-emulsions in oil-loaded monoglyceride-based particles. Colloids and Surfaces A: Physicochemical and Engineering Aspects 2006, 291 (1-3), 78-84.

56. Kulkarni, C. V.; Moinuddin, Z.; Patil-Sen, Y.; Littlefield, R.; Hood, M., Lipid-hydrogel films for sustained drug release. International Journal of Pharmaceutics 2015, 479, 416421.

57. Korsmeyer, R. W.; Gurny, R.; Doelker, E.; Buri, P.; Peppas, N. A., Mechanisms of solute release from porous hydrophilic polymers. International Journal of Pharmaceutics 1983, 15 (1), 25-35. 
58. Ritger, P. L.; Peppas, N. A., A simple equation for description of solute release I. Fickian and non-fickian release from non-swellable devices in the form of slabs, spheres, cylinders or discs. Journal of Controlled Release 1987, 5 (1), 23-36.

59. Spicer, P. T., Progress in liquid crystalline dispersions: Cubosomes. Current Opinion in Colloid \& Interface Science 2005, 10 (5-6), 274-279.

60. Zabara, A.; Mezzenga, R., Plenty of room to crystallize: swollen lipidic mesophases for improved and controlled in-meso protein crystallization. Soft Matter 2012.

61. Negrini, R.; Mezzenga, R., Diffusion, Molecular Separation, and Drug Delivery from Lipid Mesophases with Tunable Water Channels. Langmuir 2012, 28 (47), 16455-16462.

62. Clogston, J.; Caffrey, M., Controlling release from the lipidic cubic phase. Amino acids, peptides, proteins and nucleic acids. Journal of Controlled Release 2005, 107 (1), 97-111.

63. Fong, W. K.; Hanley, T.; Boyd, B. J., Stimuli responsive liquid crystals provide 'ondemand' drug delivery in vitro and in vivo. Journal of Controlled Release 2009, 135 (3), 218-226.

64. Rahanyan-Kägi, N.; Aleandri, S.; Speziale, C.; Mezzenga, R.; Landau, E. M., StimuliResponsive Lipidic Cubic Phase: Triggered Release and Sequestration of Guest Molecules. Chemistry - A European Journal 2014, 20, 1-6.

65. Angelov, B.; Angelova, A.; Garamus, V. M.; Lebas, G.; Lesieur, S.; Ollivon, M.; Funari, S. S.; Willumeit, R.; Couvreur, P., Small-Angle Neutron and X-ray Scattering from Amphiphilic Stimuli-Responsive Diamond-Type Bicontinuous Cubic Phase. J. Am. Chem. Soc. 2007, 129 (44), 13474-13479.

66. Kulkarni, C. V.; Tomšič, M.; Glatter, O., Immobilization of Nanostructured Lipid Particles in Polysaccharide Films. Langmuir 2011, 27 (15), 9541-9550.

67. Kulkarni, C. V.; Wachter, W.; Iglesias, G. R.; Engelskirchen, S.; Ahualli, S., Monoolein: A Magic Lipid? Phys Chem Chem Phys 2011, 13, 3004-3021.

68. Koerner, Megan M.; Palacio, Luis A.; Wright, Johnnie W.; Schweitzer, Kelly S.; Ray, Bruce D.; Petrache, Horia I., Electrodynamics of Lipid Membrane Interactions in the Presence of Zwitterionic Buffers. Biophysical Journal 2011, 101 (2), 362-369.

69. Peiró-Salvador, T.; Ces, O.; Templer, R. H.; Seddon, A. M., Buffers May Adversely Affect Model Lipid Membranes: A Cautionary Tale. Biochemistry 2009, 48 (47), 11149-11151. 


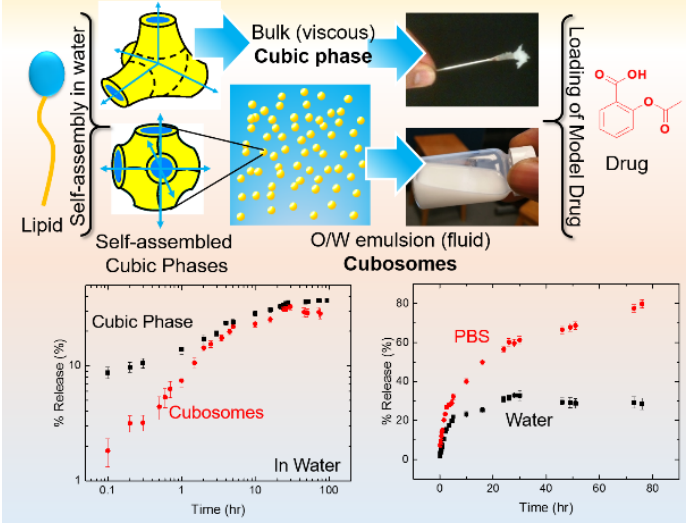

ToC Graphics 